\title{
Porcupine recognition algorithm based on Gaussian mixture background modeling
}

\author{
Shou-Hua Yu, Li-Yi Xian, Wei Yang, Tao Zou, Ling-Feng Yuan, Zhen-Guo Zhu and \\ Qing-Song Yang \\ College of Mathematics and Informatics, South China Agricultural University, \\ Guangzhou, Guangdong, China 510642 \\ E-mail:segrad@scau.edu.cn \\ www.scau.edu.cn
}

\begin{abstract}
Porcupine identification is a key part of porcupine intelligent monitoring system. This paper proposes a porcupine recognition algorithm based on Gaussian Mixture background modeling. The algorithm completes identification and feature extraction of the porcupine based on the calculation and withdrawal of image preprocessing, background modeling, foreground segmentation, contour extraction and parameter. Use the video collected in a porcupine farm to randomly screenshot 1036 frames and 1304 frames of night and daytime video image to verify the algorithm. The experimental results show that as for the image of multiple porcupines, the night recognition rate may reach $81.81 \%$. Due to the influence of daytime ray changes, shadow generates from porcupine shape and porcupine life (night activity, daytime sleeping) and the daytime correct recognition rate is only $61.41 \%$. This research provides a reference for research on the porcupine behavior recognition in intelligent monitoring system of porcupine.
\end{abstract}

Key words: Porcupine Identification; Intelligent Control; Gaussian Mixture Background Modeling; Contour Detection.

\section{Introduction}

Porcupine meat is delicious with high nutritional value and medicinal value. The hotels and restaurants in China's coastal city are favorite of the porcupine meat. The porcupine is in a sharp increase in demand, but the price is expensive. But the wild porcupine is three-level national protected animal, which is in increasing depletion. Countries have banned hunting and vigorously promoted artificial domestication. The wild porcupine is used to wild habit (violent, Eccentric and impatient and observant) due to long-term wild status, so it is inconvenient to directly observe the behavior of porcupine. The manual observation labor intensity is strengthened due to less activity in daytime and foraging at night [1]. These factors bring huge difficulty to the artificial breeding, thus the real-time monitoring of porcupine cannot be guaranteed. 
Through literature analysis and field investigation, it is found that the monitoring in artificial porcupine houses relies on the observation of aquaculture management personnel by turns and the rich experience in the analysis of the status. The high labor intensity (porcupines mostly behave at night) and low work efficiency mode requires highly in the observation personnel. Porcupine breeding benefit is high [1], but China is still in the stage of the rise of porcupine breeding and the breeding scale is not big. Currently, there is less research on porcupine breeding monitoring system. No relevant article is found in CNKI in China with "porcupine" and "monitoring" as key word. Therefore, the research on porcupine breeding monitoring is important and necessary to deeply understanding porcupine habits and improving production efficiency of porcupine breeding. To achieve real-time intelligent monitoring of porcupine, the first problem is to recognize the porcupine. The paper proposes a porcupine recognition algorithm based on Gaussian Mixture background modeling according to the characteristics of porcupine houses environment, porcupine's living habits and porcupine's color features.

\section{Algorithm Flow Design}

In the process of target detection in intelligent monitoring system in the porcupine houses, the arbitrariness of irregular porcupine activities determines the irregularity of shape [2]. It is difficult to obtain the shape of individual porcupine. It is very difficult to separate the porcupine and background and difficult to detect multiple porcupines due to porcupine's living environment, living habit (rest in daytime, behaving at night) and the complex texture characteristics of individual porcupine. But the background is static, the porcupine is moving, and porcupine size is different, so this research takes use of its movement features when testing multiple porcupines and the detection bases on Gaussian mixture background modeling. The algorithm detection process is shown as follows: (1) image preprocessing; (2) the background modeling and foreground segmentation; (3) contour search; (4) the calculation and extraction of contour parameters; (5) the porcupine identification and feature extraction. The specific algorithm flow is as shown in figure 1. 


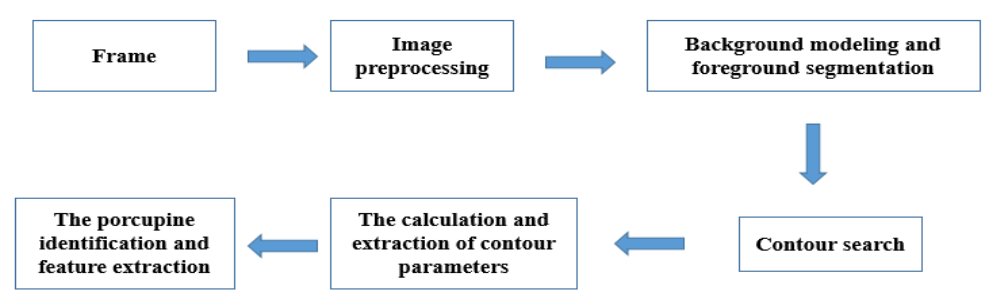

Fig. 1 Algorithm flow chart

\section{Foreground Segmentation and Background Modeling}

Everything that is visible to the naked eye can be called a background. To spend more time in understanding the object, the object which can provide the necessary information is called the foreground. In this study, the porcupine is prospect and the real need is to analyze the porcupine. Porcupine living environment is the background. In this study, the purpose is to detect and analyze porcupine, but porcupine houses may cause interference in extracting the porcupine. Therefore, the foreground segmentation and background modeling is distinguishing the porcupine and background from the video image, excluding other interferences outside the porcupine.

There are a variety of methods, such as the method based on Single Gaussian Modeling, the method based on Mixture of Gaussian model, background subtraction method based on statistics, modeling method based on Codebook and so on [3]. Choosing the right method for the experimental results will be decisive. Analyze with the original video. In the porcupine houses environment, the porcupine is close to the background color. This will lead to poor results based on color features and texture features. Subsequent experiments also verify this. For the moving objects, the movement characteristics may better separate the moving objects from background. The Single Gaussian Modeling and Gaussian Mixture Background Modeling can effectively extract the contour of porcupine, and then obtain the features and motion information of the porcupine.

The paper selects four background modeling methods according to the previous research and analysis, which is respectively Mixture of Gaussian Model, Frame Difference Method, Codebook and Fixed Background Method. After research and analysis, Mixture of Gaussian Model, Frame Difference Method and Codebook are used in the experiment. 


\subsection{Fixed background method}

In this algorithm, an image is used as the background, and then the foreground is detected on the basis of this. The core formula is $\mid$ frame(i)-background(i) $\mid>T h$. Distinguish between foreground and background by setting specific thresholds. As the background is a fixed setting image, here is the introduction of four issues: Illumination variation, camera jitter, high frequency oscillation background, moving to stationary object. The algorithm is simple and easy to implement, but the camera must be absolutely stationary, and does not adapt to the changes in illumination. Due to the analysis of the porcupine behavior, it needs 24-hour real-time monitoring, during which the light will change with time, and the shaking of the camera can cause greater interference, so the fixed background method is excluded.

\subsection{Frame difference method}

Frame difference method [4] is one method of background difference method, but it requires no modeling. Because the background model is the image of former frame, so the speed is very fast. But it is not sensitive to the luminance changing slowly. As shown in the experimental results, the Unicom region in the forecast figure obtained with frame difference method is too scattered, which causes the contour search divides the Porcupine into different contours, so the method is not used.

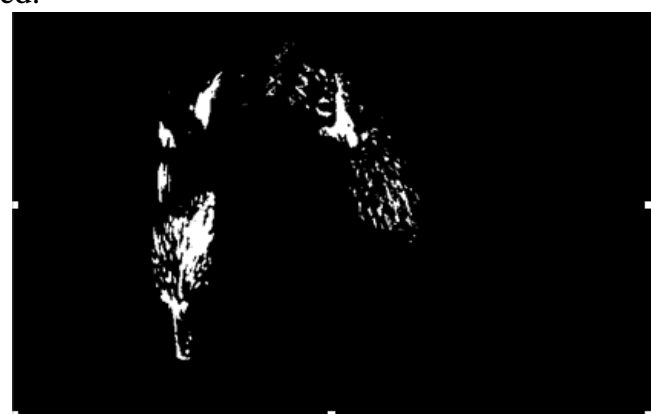

Fig. 2 Experimental effect diagram of frame difference method

\subsection{Codebook}

Codebook [5] algorithm uses quantization technique to construct background model from long-term observation sequence. It may establish a codebook for each pixel. Each codebook contains a group of codes. This model can well deal with the impact of time fluctuations, but it requires consuming a large amount of memory. As shown in the experimental effect, the closure zone of forecast image 
is more compact that frame difference method, but there still is scattered zone.

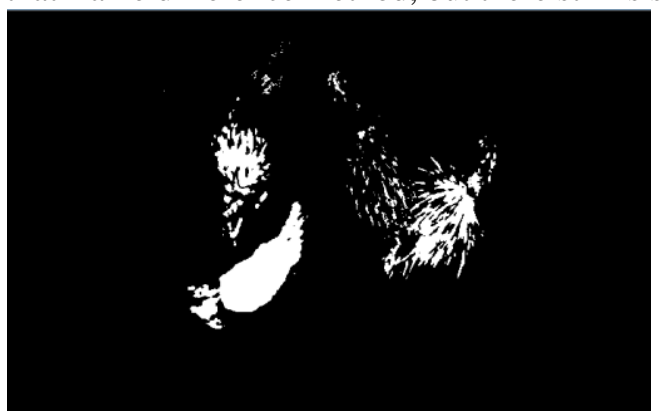

Fig. 3 Experimental effect diagram of Codebook

\subsection{Mixture of Gaussian Model}

Gaussian model is such a model that use the Gaussian probability density function (normal distribution curve) to accurately quantify things and decompose a thing into a number of functions based on the Gaussian probability density (normal distribution curve).

Mixture of Gaussian Model uses K (usually 3 to 5) Gaussian models to represent the features of each pixel point in the diagram [6]. Update Mixture of Gaussian Model after obtaining a new frame and use each pixel in current figure to be matched with Mixture of Gaussian Model. If successful, the pixel will be judged as background pixel, or it will be forecast pixel. The whole Gaussian model is determined by variance and mean. As for the learning on mean and variance, use different learning mechanisms to directly influence the model accuracy and stability.

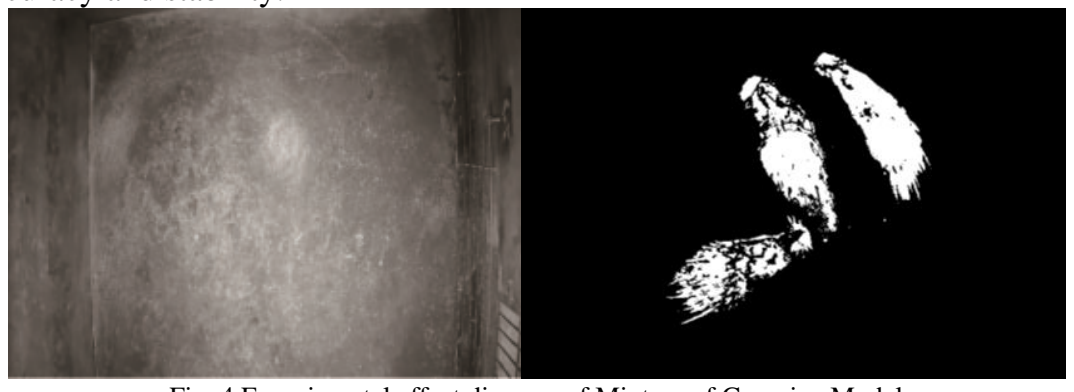

Fig. 4 Experimental effect diagram of Mixture of Gaussian Model

The experiment found that the fixed background requires high video quality, and it cannot reach the requirement. The porcupine effect is poor and information is lost seriously detected by frame difference method. Although Codebook detects porcupine volume completely, but it may produce ghost and 
consume a lot of memory. It seriously influences the porcupine recognition. As shown in figure 4, Mixture of Gaussian Model may better withdraw the background and distinguish porcupine and porcupine house background, and the operation speed and internal memory consumption are in the reasonable scope. Upon comprehensive considerations, giving up the fixed background method which cannot meet requirements in the recording technology and the frame difference method with poor background modeling effect, and the codebook with high consumption in internal memory, the background modeling method based on Mixture of Gaussian Model will be used for background modeling and forecast segment. On this basis, further conduct research improvement combining with the features of Porcupine and its living environment.

\section{Porcupine Recognition based on Mixture of Gaussian Background Modeling}

\subsection{Image preprocessing}

For each frame, the preprocessing is required to facilitate the subsequent processing of Mixture of Gaussian Model. In the image preprocessing stage, the operations are: RGB to greyscale transfer, eliminate the Gaussian noise in Gaussian filter and median filtering noise reduction.

\subsubsection{RGB to grey levelimage transfer}

Grey image is the image of each pixel with only one sample color. Different from the black and white image, there are many levels of color depth between black and white. In this study, the image processing does not need to deal with the three components of the color image. Converting the original image into a grey image can greatly reduce the original data of the image, so as to reduce the computational complexity and quantity and speed up the processing speed.

\subsubsection{Image denoising}

Gauss filter is a linear smoothing filter, which can eliminate the Gauss noise, and it is widely used in image processing noise reduction process. The principle is to process the weighted average of the whole image, each pixel value of the pixel is obtained by itself and the other pixels in the field by the weighted average. In this study, because the original image obtained by the porcupine house environment contains a lot of noise, and will have great effect on the subsequent processing, Gaussian filtering method is used to preprocess the image, in order to achieve the purpose of noise reduction. 
Median filtering is a typical and commonly used nonlinear filtering technology, and it is also the most commonly used technology in image processing. The basic idea is to use the median value of grey value in the neighborhood pixel points to replace the grey level of the pixel value, namely the value of one point in digital picture or digital sequence is replaced by the median value of each point in neighborhood field, in order to make the surrounding pixel value near to the actual value and eliminate the isolate noise points. It is very effective in smooth pulse noise and impulse noise, and it may protect the sharp edge of image. Weighted median filter can improve the edge signal to maintain the effect of median filter. Because the median value filtering may overcome the fuzzy details caused by linear filter in certain case, and also it is very effective to filter pulse interference and image scanning noise and is commonly used in protecting edge information. It can effectively filter out the high frequency noise points and keep the complete face target, but it cannot suppress the low frequency background.

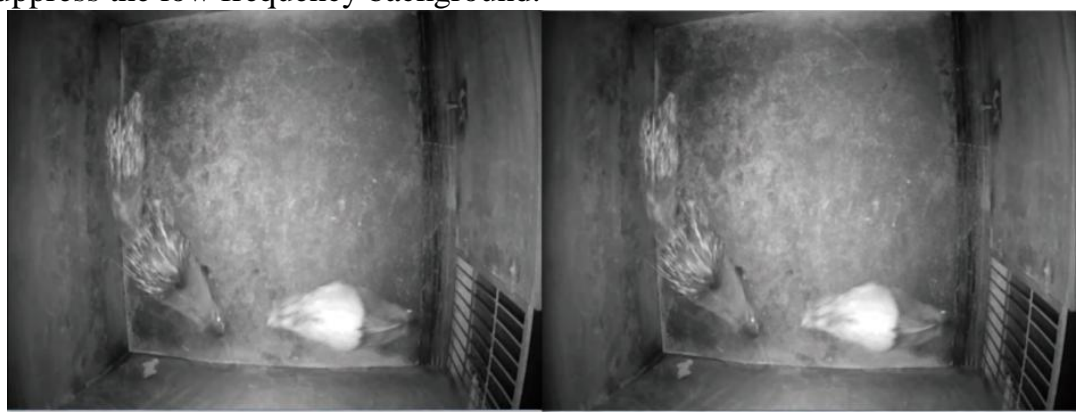

Fig. 5 Original figure effect (left) and preprocessing effect (right)

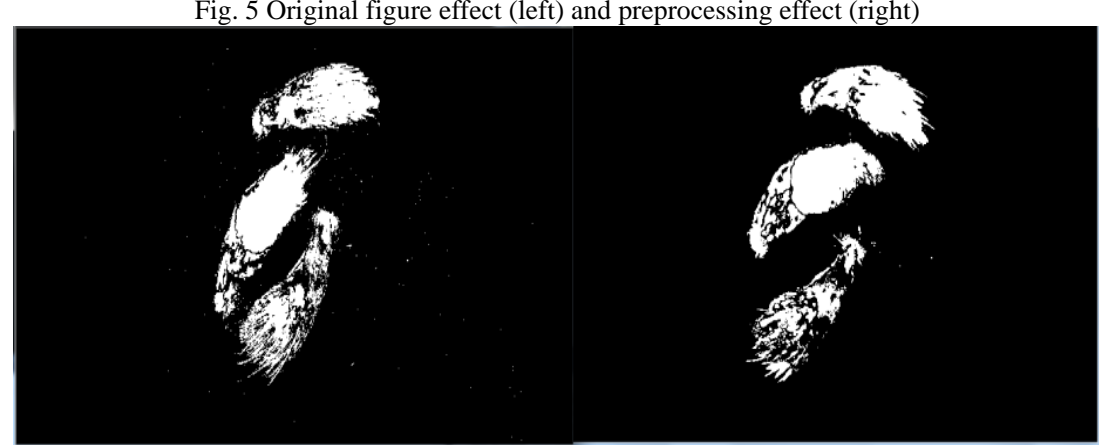

Fig. 6 Original figure mask effect (left) and post-processing mask effect (right)

The research combines the advantages and disadvantages of Gaussian filtering and medium filtering. Firstly, take use of color space conversion to change each frame of RGB image to grey image, and the use Gaussian filtering 
in low pass filtering operation and Gaussian noise reduction [7]. And then take use of medium filtering to filter high-frequency noise points and maintain the complete targets. Figure 5 and 6 show the image and mask image before or after processing. As shown in mask image, the amount of noise points will be reduced obviously after preprocessing, making mask image more clear and facilitating the subsequent outline search.

\subsection{Mixture of Gaussian Background Model}

Gaussian Background Model regards the grey level of one pixel on video or image sequence as one random process $X$, and supposes the grey level is subject to Gaussian distribution [8]. The method can better adapt to the complex background image, and accurately separate forecast from background. The modeling method of indicating background with one Gaussian distribution mode is called Single distributed Gaussian background. Several Gaussian distribution models is used to weigh and model Mixture of Gaussian Background Model.

In the image of changing to grey level, the occurrence frequency of each grey level conforms to Gaussian distribution. In case of high difference between the forecast and background grey value in the mage, the Grey histogram in the image is in doublet shape, one is corresponding forecast and the other is corresponding background. As for the complex images, there are multiple peaks in histogram. The purpose of Gaussian modeling is to take use of several Gaussian distributions to divide the images. The pixels conforming to Gaussian distribution of background is called background, or it is called forecast on the contrary. The background may be divided from the image.

\subsection{1. $\quad$ Single distributed Gaussian Background Modeling}

Single distributed Gaussian background modeling applies to the condition of single peak distribution and constant background. The color distribution of each pixel is indicated with single Gaussian distribution function. As for the brightness $\mathrm{Ib} \sim \mathrm{N}(\mathrm{u}, \mathrm{d})$ of image to be detected $\mathrm{A}(\mathrm{x}, \mathrm{y})$, we will calculate the mean $\mu$ and variance $\delta$ of each point. If $\operatorname{Exp}\left(-(\operatorname{IG}(x, y)-u(x, y))^{\wedge} 2 /\left(2 * d^{\wedge} 2\right)\right)>\mathrm{T},(x, y)$ is deemed as background and it is forecast on the contrary[9].

$$
\text { Image }=\left\{\begin{array}{l}
\text { background } \operatorname{Exp}\left(-(I(x, y)-\mu(x, y))^{2} /\left(2 \times \sigma^{2}\right)\right)>T \\
\text { foreground otherwise }
\end{array}\right.
$$

\subsubsection{Mixture of Gaussian Background Modeling}

Mixed Gauss background model is a weighted sum of the finite Gauss's 
functions. It may describe multi-peak status of pixel and apply to the correct modeling of Light gradient (the main change of porcupine house background), tree swing and other complex background[10]. It is the improvement of single Gaussian background model and an effective method of complex background modeling.

In the mixture of Gaussian model, the color information between pixels is irrelevant. Each pixel may be separated and processed. In one video, the pixels generated from new frame still use the model established and updated in the last frame image for background and forecast judgment.

If $\mathrm{I}(\mathrm{x}, \mathrm{y}, \mathrm{t})$ indicates the pixel value of $(\mathrm{x}, \mathrm{y})$ at $\mathrm{t}$, the mixture of Gaussian background model established in the pixel may be indicated as[11]:

$$
\mathrm{P}(\mathrm{I}(\mathrm{x}, \mathrm{y}, \mathrm{y}))=\sum_{i=1}^{K} \omega_{\mathrm{i}}^{\mathrm{t}} \cdot \eta_{\mathrm{i}}\left(\mathrm{I}(\mathrm{t}), \mu_{i}^{\mathrm{t}}, \sigma_{i}^{\mathrm{t}}\right)
$$

Where, $\mathrm{K}$ is the number of Gaussian distribution model and is the weight of i Gaussian distribution at $t, \eta$ is the possibility density function of Gaussian distribution.

$\mathrm{K}$ Gaussian distribution models corresponding to each pixel is sorted from high to low based on $\omega / \sigma$. Select $n$ Gaussian distribution as the background model.

Conditions of successful matching

If the difference between grey level $\mathrm{I}(\mathrm{x}, \mathrm{y}, \mathrm{t})$ of certain pixel $(\mathrm{x}, \mathrm{y})$ and the mean of $\mathrm{m}(\mathrm{m} \leq \mathrm{n})$ Gaussian distribution in background model meets the following formula, the matching is successful, and the pixel is deemed as background pixel.

$$
\left|\mathrm{I}(\mathrm{x}, y, t)-\mu_{m}^{t}\right|<\lambda \cdot \sigma_{m}^{t}
$$

Update background parameters:

If $\mathrm{I}(\mathrm{x}, \mathrm{y}, \mathrm{t})$ of pixel $(\mathrm{x}, \mathrm{y})$ in current frame and $\mathrm{i}$ Gaussian model are matched successfully, update the mean and variance of Gaussian distribution:

$$
\begin{gathered}
\mu_{\mathrm{i}}^{t+1}=(1-\beta) \cdot \mu_{t}^{i}+\beta \cdot I(t+1) \\
\left(\sigma_{i}^{t+1}\right)^{2}=(1-\beta)\left(\sigma_{i}^{t}\right)^{2}+\beta\left(I(t+1)-\mu_{i}^{t+1}\right)^{T}\left(I(t+1)-\mu_{i}^{t+1}\right)
\end{gathered}
$$

$\alpha$ is updating ate and the scope is $[0,1]$

$\beta$ is: 


$$
\beta=\alpha \cdot \eta\left(\mathrm{I}(t) \mid \mu_{i}, \sigma_{i}\right)
$$

The unmatched Gaussian distribution mode requires no updating mean and variance. At $\mathrm{t}+1$, update the weight of $\mathrm{K}$ Gaussian distribution models:

$$
\omega_{i}^{t+1}=(1-\alpha) \cdot \omega_{\mathrm{i}}^{\mathrm{t}}+\alpha \cdot D_{i}^{t}(I \leq K)
$$

Where, Gaussian distribution of successful matching $\mathrm{D}_{\mathrm{i}}^{\mathrm{t}}=1$, the rest $\mathrm{K}-1$ Gaussian distribution $\mathrm{D}_{\mathrm{i}}^{\mathrm{t}}=0$.

After updating, sort K Gaussian models according to the weight size.

Summarize the algorithm process as follows:

(1)Compare each pixel $\mathrm{X}_{\mathrm{t}}$ of current frame with $\mathrm{K}$ Gaussian distribution;

(2)If the matched background Gaussian distribution is found, the pixel point is background or it will be indicated as forecast.

(3)Update K model currently;

(4)Return to step (1) until processing the final frame of video

\subsection{3. $\quad \alpha$ Parameter selection analysis}

The learning rate $\alpha$ of mixture of Gaussian background model will influence the modeling effect. When $\alpha$ is 0.0001 (figure 7), the leftover time of forecast ghost is long and inconvenient to recognize the forecast. The background will mix Porcupine prospects. When $\alpha$ is 0.01 (figure 9), the forecast image updating is too fast. Any transient stillness of target object will make the forecast lost. The advantage is that it may obtain the background diagram with good effect. When $\alpha$ is 0.001 (figure 8), the Porcupine prospect may be obtained better. The defect is the background diagram may mix the ghost of Porcupine prospect. To obtain the prospect with better effect, $\alpha$ may take the value 0.001 ; to obtain the background with better effect, $\alpha$ may take the value 0.01 .

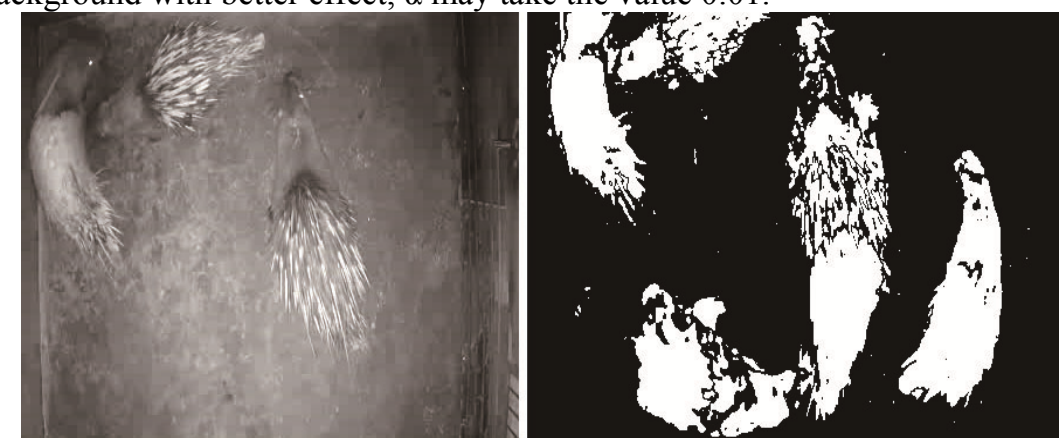

Fig. 7 Gaussian background modeling diagram and mask diagram when the learning rate is 0.0001 

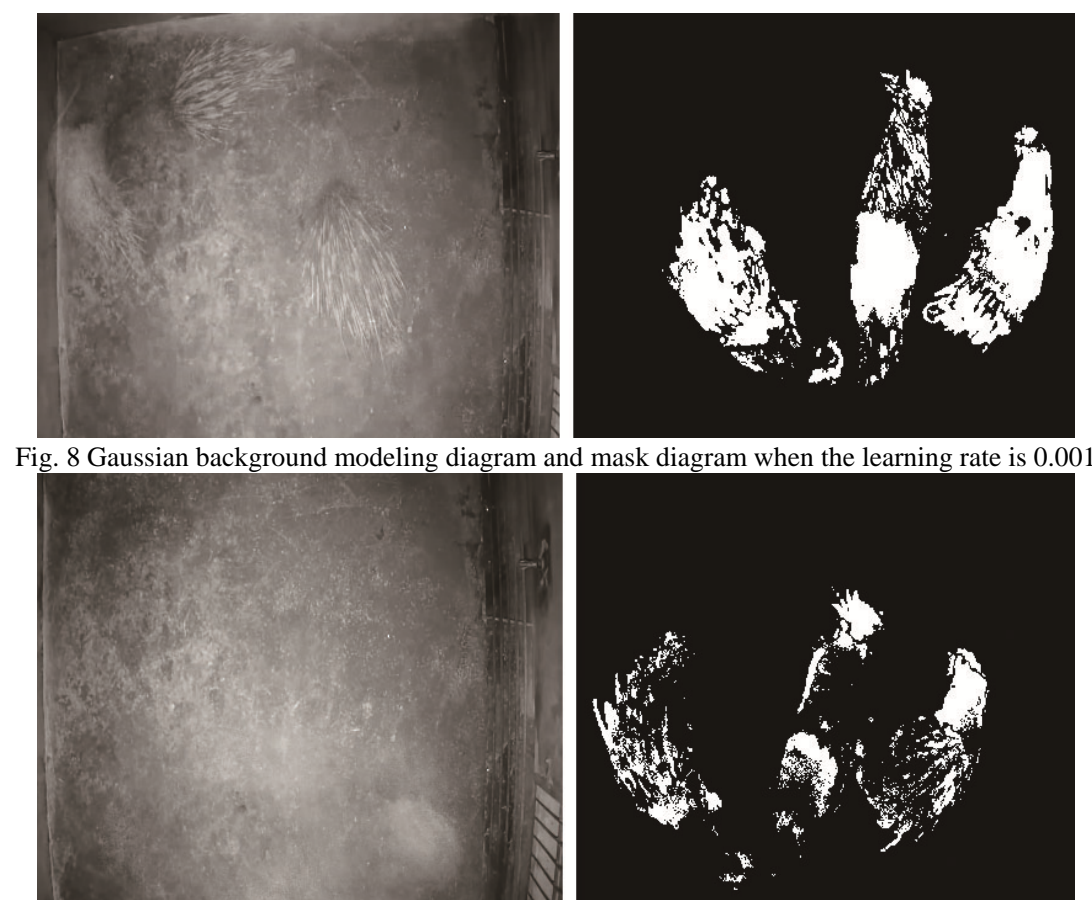

Fig. 9 Gaussian background modeling diagram and mask diagram when the learning rate is 0.01

\subsection{Contour Search}

The image analysis based on contents is withdrawing the representative features in the image. Several elements which may embody the features are Line, outline, block. In order to further study, we detect and withdraw porcupine contour based on the Gaussian background modeling, in order to obtain the contour perimeter porcupine [12], area and $\mathrm{Hu}$ moment, and then identify the number of porcupine video images, determine the location of the porcupine and provide the initial position tracking algorithm.

Because we have obtained the image with prospect (namely porcupine) in Gaussian background modeling stage, we will take the image in each fame as the input of outline detection function to withdrawn the outline of porcupine. The obtained outline will be drawn on the non-processing original video image for comparison. Observe whether the outline is matched with porcupine in original video image to judge the algorithm precision and accuracy. 


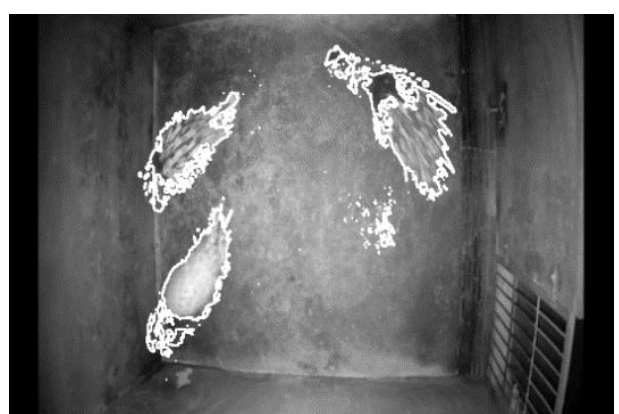

Fig. 10 Schematic diagram of outline detection

\subsection{Porcupine Identification}

Because the following research is the porcupine tracking and behavior recognition, it is required to get the Porcupine number, outline area Hu moment and initial position in the video image. We will process with the obtained outline to obtain and store the required data. The research achieves Porcupine identification and definition through two steps: Preliminary outline screening and contour comparison. At the initial stage, calculate the area of all outlines in each frame, and compare the solved outline area with the preset threshold. If larger than the threshold, the outline size is deemed as similar to Porcupine. Record the outline and shape features lager than threshold, such as area, perimeter, Hu moment, etc. for further comparison. The threshold setting comes from the observation and evaluation in experimental process. multiple experiments and corrections may guarantee Porcupine outline above threshold. The irrelevant outline shall be prevented from comparison to reduce the memory and time consumption. In outline comparison stage, the data outline stored in initial stage will be compared with the presetting Porcupine outline model. If the parameters are consistent with the model, the outline will be deemed as Porcupine. Count and store all feature parameters obtained in this stage for reference.

\section{Experimental Analysis}

Select 1036 frames of night video images and 1304 frames of daytime video images from the image of Porcupine house for verification. Mark the identification area in output image with red. As for night video image identification, the identification may be deemed as success when meeting the following conditions: 1)Coincidence rate between identification area and Porcupine area exceeds $80 \%$;2) Coincidence rate with wrong identification area 
is less than 20\%; 3) Coincidence rate between identification area and Porcupine area exceeds $10 \%$. In 1036frames, the former 200 frames are used to learn algorithm and the later 836 frames are used in detection. In case of containing multiple Porcupines, correctly identify 684 frames (figure 11) and wrongly identify 152 frames. The correctness of identification reaches $81.81 \%$. It meets the expect effect. The daytime video images will be identified due to the daytime ray changes, shadow of Porcupine and Porcupine life (sleeping in daytime and moving at night). The identification may be deemed as success when meeting the following conditions: 1)Coincidence rate between identification area and Porcupine area exceeds $60 \%$; 2) Coincidence rate with wrong identification area is less than $40 \%$; 3 ) Coincidence rate between identification area and Porcupine area exceeds 20\%. The same as the night identification. In 1304 frames, the former 200 frames are used to learn algorithm and the later 1104 frames are used in detection. In case of containing multiple Porcupines, correctly identify 678 frames (figure 12) and wrongly identify 426 frames (figure 13). The correctness of identification reaches $61.41 \%$.

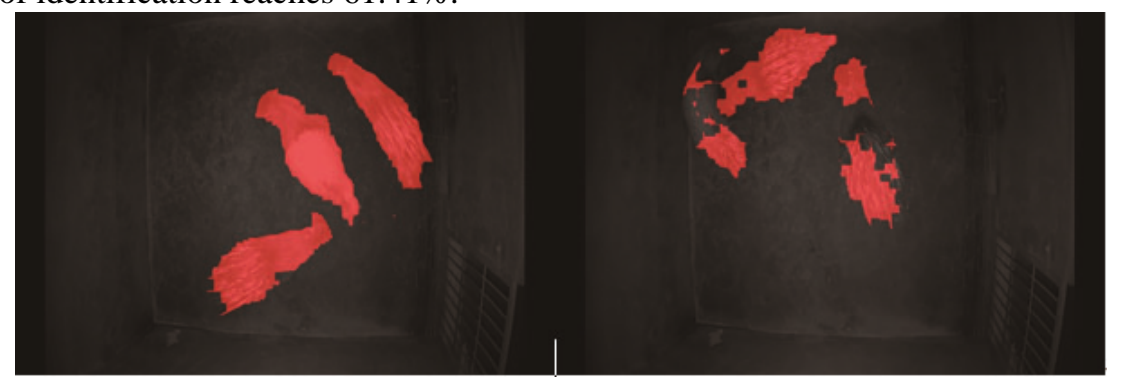

Fig. 11 Example of correct identification of night image (left) and wrong identification (right)

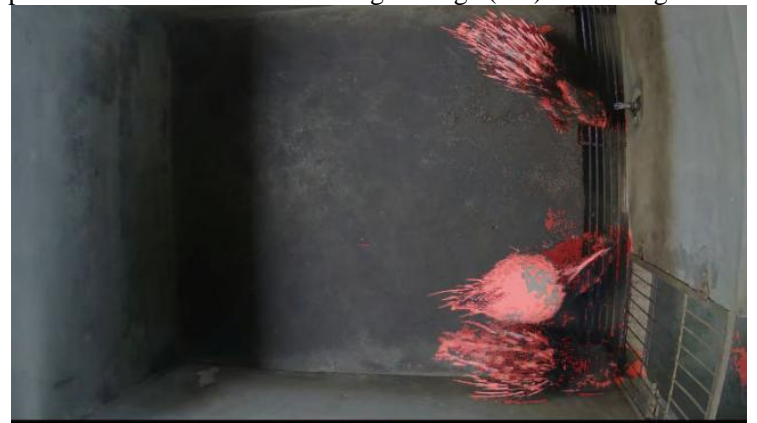

Fig. 12 Example of correct identification of daytime image 


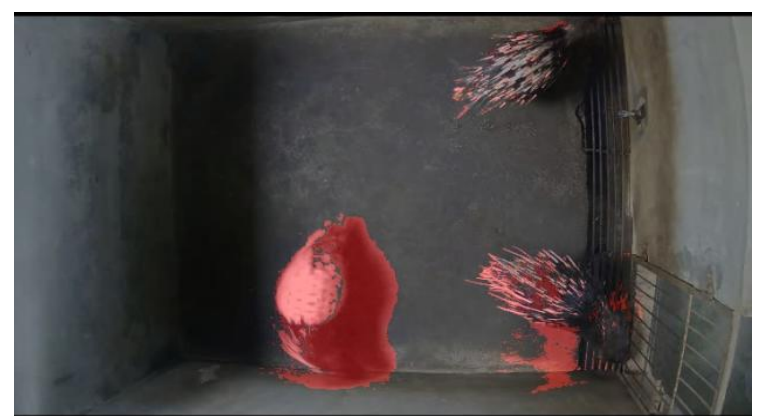

Fig. 13 Example of influence of ray changes and porcupine shadow

\section{Conclusion}

This algorithm proposes porcupine identification method which is applicable to porcupine house intelligent monitoring system based on the mixture of Gaussian modeling algorithm. The algorithm completes identification and feature extraction of the porcupine based on the calculation and withdrawal of image preprocessing, background modeling, foreground segmentation, contour extraction and parameter. The algorithm is verified with 1036 frames of night video image and 1304 frames of daytime video image screenshot in the video of Guangzhou ConghuaShuguang Porcupine Agricultural Farms. The results show that the correct identification rate of night scene of multiple Porcupines reaches $81.81 \%$, which meets the requirement of Porcupine behavior identification in Porcupine house intelligent monitoring system. The deficiency of the research lies in that: the number of outlines is less than the actual number of Porcupine when several Porcupines are connected and Porcupines do not move relatively. It is easy to cause large derivation in the calculation number due to the daytime ray changes, shadow of Porcupine and Porcupine life (sleeping in daytime and moving at night), the identification effect of video image on daytime scene is inferior to that at night. There still is large improvement space.

\section{Acknowledgments}

This work was supported by Science and Technoogy Planning of Guangdong Provine under contract 2012A020602043 and College students innovation and entrepreneurship training program of South China Agricultural University under contract 201510564280.

\section{References}

1. Xia Qingshui, High Breeding Efficiency of Porcupine, New Rural 
Technology,1(2016),p. 47. (In Chinese).

2. Chen Jihong, Yu Shouhua and Qu Jingying, Research on pig recognition algorithm for intelligent monitoring system of pigpen, Guangdong Agricultural Sciences, 10(2011),pp.151-152. (In Chinese).

3. Li Xiaopeng, Yan Yan and Zhang Yujin, Analysis and Comparison of Background Modeling Methods, The Thirteenth National Conference on Image And Graphics(Nanjing, Jiangsu, China,2006),p. 5. (In Chinese).

4. Liang Zhiyong, Research of intelligent monitoring system based on least frame difference method for image recognition, Mater's thesis, Guangdong University of Technology(2005),p.65. (In Chinese).

5. Ye Yong, Guang Yepeng and Li Jingjing,Foreground Target Detection based on Gaussian Mixture Model and Codebook Algorithm, Computer Engineering, 5(2012),pp.1-4. (In Chinese).

6. Feng $\mathrm{Pu}$, Research on Mixture of Gaussian Background Modeling Algorithm Based On Texture Feature,Computer Technology And Development,,4(2016),pp.1-6. (In Chinese).

7. Zhou Shanshan, Chai Jinguang, Research on Filtering Algorithm of Image Preprocessing,Science Technology and Engineering, 13(2009),p.3830. (In Chinese).

8. Bouwmans T. Traditional and recent approaches in background modeling for foreground detection: An overview, Computer Science Review, s 11-12 (2014),p.40.

9. Kang Xiaojing, Wu Jin: Object Detecting Technology Based on Gauss Background Modeling, Chinese Journal of Liquid Crystals and Displays, 3(2010),pp.454-459. (In Chinese).

10. Chris Stauffer, and W.E.L. Grimson. Adaptive Background Mixture Models for Real-Time Tracking, in Proceedings / CVPR, IEEE Computer Society Conference on Computer Vision and Pattern Recognition, IEEE Computer Society Conference on Computer Vision and Pattern Recognition 2(1999):2246.

11. Fradi H, Dugelay J. Robust foreground segmentation using improved Gaussian Mixture Model and optical flow, International Conference on Informatics, Electronics \& Vision. IEEE, (Dhaka, Bangladesh 2012),pp.249-250.

12. Liu Huiying and Wang Xiaobo: Vehicle contour detection based on OpenCV, Science Technology and Engineering, 12(2010),pp.2987-2991. (In Chinese). 